\title{
Perfil da prescrição de medicamentos para o diabetes mellitus tipo 2 de um município paulista
}

\author{
Prescription profile of medicines for type 2 diabetes mellitus of the municipality \\ of the state of São Paulo
}

\author{
Julieta Ueta ${ }^{a}$, Sílvia Ferreira Lima Cavalheirob ${ }^{b}$ Anali Duarte Ortigoso Carbic, Daniela Yumi Takatad, \\ Laercio Joel Franco ${ }^{e}$, Rinaldo Eduardo Machado de Oliveira ${ }^{\dagger}$ \\ a Farmacêutica. Pós-doutora em Bioquímica. Faculdade de Ciências Farmacêuticas de Ribeirão Preto da Universidade de São Paulo. \\ ${ }^{\text {b}}$ Farmacêutica. Doutora em Ciências Farmacêuticas. Faculdade de Ciências Farmacêuticas da Universidade Estadual Paulista Júlio de Mesquita Filho - Campus Araraquara. \\ cFarmacêutica. Especialista em Saúde Coletiva. Prefeitura Municipal de Limeira. \\ dFarmacêutica. Especialista em Saúde Coletiva. Instituto Butantan. \\ eMédico Endocrinologista. Pós-doutor em Epidemiologia Clínica. Faculdade de Medicina de Ribeirão Preto da Universidade de São Paulo. \\ ' Farmacêutico. Mestre em Ciências. Faculdade de Medicina de Ribeirão Preto da Universidade de São Paulo. \\ Fonte de Financiamento: Ministério da Saúde.
}

RESUMO

Objetivo: Analisar as prescrições de medicamentos para o diabetes mellitus tipo 2 (DM2) considerando as atuais recomendações clínicas, em Unidades de Saúde da Família de Ribeirão Preto, São Paulo.

Materiais e Métodos: Trata-se de um estudo retrospectivo realizado por meio da análise de prontuários das pessoas com DM2 usuárias de medicamentos. O tamanho da amostra calculado foi de 150 participantes. Foram obtidos os dados sociodemográficos, clínicos, medicamentos prescritos e resultado do teste laboratorial de hemoglobina glicada.

Resultados: A maioria dos indivíduos pertencia ao sexo feminino (61,3\%) e a média de idade foi de 66,4 anos. O controle glicêmico apresentou-se inadequado em 75,3\% dos participantes, com hemoglobina glicada média de 8,8\%. A média de medicamentos prescritos para o tratamento do DM2 foi de 1,7. Nas pessoas com idade inferior a 60 anos predominou a prescrição de sulfonilureias. Dentre os idosos, predominou a prescrição de metformina, seguido pelas sulfonilureias e insulina, respectivamente. Observou-se associação entre o controle glicêmico inadequado e pessoas em uso de terapia oral combinada $[O R=0,32(I C 95 \% 0,12 ; 0,80)]$ e insulinoterapia isolada ou em associação com antidiabético oral $[\mathrm{OR}=0,05$ (IC95\% 0,01; 0,18)]. Além disso, evidenciou-se associação entre o controle glicêmico inadequado e pessoas com dois $[\mathrm{OR}=0,3(\mathrm{IC} 95 \% 0,13 ; 0,67)]$ ou três $[\mathrm{OR}=0,08(\mathrm{IC} 95 \% 0,01 ; 0,70)]$ medicamentos prescritos

Conclusão: $O$ controle glicêmico apresentou-se inadequado na maioria dos participantes. A monoterapia foi o tratamento mais frequente, embora, uma parcela considerável estudada usasse sulfonilureia em monoterapia, o que está em desacordo com as atuais diretrizes preconizadas para o tratamento do DM2.

Palavras-chave: diabetes mellitus; doença crônica; tratamento farmacológico; prescrições de medicamentos; estratégia saúde da família.

Objective: To analyze the prescriptions of drugs for type 2 diabetes mellitus (T2DM) considering current clinical recommendations, in Family Health Units of Ribeirão Preto, São Paulo.

Materials and Methods: This is a retrospective study carried out through the analysis of medical records of individuals with T2DM who are using medication. The calculated sample size was 150 participants. Socio-demographic, clinical data, prescription of drugs and laboratory test results for glycated hemoglobin were obtained.

Results: The majority of individuals were female (61.3\%) and the mean age was 66.4 years. The glycemic control was inadequate in $75.3 \%$ of the participants, with an average glycemic hemoglobin of $8.8 \%$. The mean number of drugs prescribed for T2DM treatment was 1.7. In individuals under the age of 60, the prescription of sulfonylureas predominated. Among the elderly, prescription of metformin predominated, followed by sulphonylureas and insulin, respectively. There was an association between inadequate glycemic control and individuals using oral combined therapy $[O R=0.32(95 \% \mathrm{Cl} 0.12 ; 0.80)]$ and insulin therapy alone or in combination with oral antidiabetic $[O R=0.05(95 \% \mathrm{Cl} 0.01 ; 0.18)]$. In addition, there was an association between inadequate glycemic control and individuals with two $[\mathrm{OR}=0.3(95 \% \mathrm{Cl} 0.13 ; 0.67)]$ or three $[\mathrm{OR}=0.08(95 \% \mathrm{Cl}: 0.01 ; 0.70)]$ prescribed medicines.

Conclusion: The glycemic control was inadequate in most participants. Monotherapy was the most frequent treatment, although a considerable proportion of patients used sulfonylurea monotherapy, which is not in accordance with current guidelines for the treatment of T2DM.

Keywords: diabetes mellitus; choric disease; drug therapy; drug prescriptions; family health strategy. 


\section{INTRODUÇÃO}

O diabetes mellitus (DM) é um conjunto de alterações metabólicas, de origens heterogêneas, caracterizado por hiperglicemia e distúrbios no metabolismo de carboidratos, resultantes de problemas na secreção e/ou ação da insulina ${ }^{1}$. Dentre os tipos de diabetes, o diabetes mellitus tipo 2 (DM2) corresponde de 90 a 95\% dos casos, no qual, há defeitos na ação e secreção da insulina, bem como, na regulação da produção hepática de glicose² .

O número de pessoas com DM é preocupante e configura-se em um dos principais problemas de saúde pública $^{1-2}$. Dados do estudo de vigilância de fatores de risco e proteção para doenças crônicas por inquérito telefônico (Vigitel) de 2016 mostrou que 8,9\% dos participantes referiram diagnóstico médico de diabetes, ao passo que, esta proporção era de 5,5\% em 2006. $\mathrm{Na}$ última década, estima-se um aumento de $61,8 \%$ na prevalência do DM no Brasil. Este crescimento é atribuído às mudanças na estrutura etária, nutricional e social da população ${ }^{3}$.

O diabetes é considerado uma condição sensível à atenção primária, em que o manejo adequado evita hospitalizações e mortes por complicações cardiovasculares e cerebrovasculares. Assim, a Estratégia Saúde da Família (ESF) tem um papel fundamental no diagnóstico, tratamento e controle do $\mathrm{DM}^{5}$.

No tratamento do diabetes são propostas ações educativas, mudanças no estilo de vida e principalmente o uso de medicamentos. A escolha da farmacoterapia, visando o controle glicêmico, dependerá da capacidade secretória do pâncreas, doenças associadas ao diabetes e características biopsicossociais dos indivíduos ${ }^{1,2}$. Na utilização de medicamentos, as equipes das Unidades de Saúde da Família (USFs) têm grande importância, uma vez que, colaboram na análise dos aspectos relacionados à obtenção dos medicamentos, adesão, problemas farmacoterapêuticos e a evolução da doença ${ }^{4}$.

As atuais diretrizes do DM2 recomendam o uso de medicamentos imediatamente após o diagnóstico clínico da doença e metformina sendo o fármaco de escolha para iniciar o tratamento ${ }^{1,2}$. Neste sentido, o objetivo deste estudo foi analisar as prescrições de medicamentos para o DM2 considerando as atuais recomendações clínicas, em USFs de Ribeirão Preto, São Paulo. Os dados aqui apresentados possibilitam o conhecimento da realidade local, subsidiam o planejamento de ações efetivas e servem de apoio para avaliar o impacto de futuras intervenções relacionadas à farmacoterapia do DM2 na ESF.

\section{MATERIAIS E MÉTODOS}

Trata-se de um estudo retrospectivo realizado por meio da consulta e análise de prontuários das pessoas com DM2 usuárias de medicamentos cadastradas em quatro USFs do Distrito Sanitário Oeste do município de Ribeirão Preto, São Paulo, no período de julho a dezembro do ano 2014. Durante o planejamento do estudo havia cinco USFs gerenciadas pelo Departamento de Medicina Social da Faculdade de Medicina de Ribeirão Preto da Universidade de São Paulo em parceria com a Secretaria Municipal de Saúde de Ribeirão Preto, São Paulo. Destas unidades, quatro concordaram em participar do estudo.

Foram incluídas no estudo as pessoas com diagnóstico médico de DM2 descrito no prontuário, idade igual ou superior a 30 anos, ambos os sexos, informações completas sobre os medicamentos prescritos e realização de consultas nas USFs no ano de 2014. Foram excluídas as pessoas com DM2 que não eram acompanhadas pelas equipes das USFs e indivíduos com histórico de hospitalização ou cirurgia nos três meses que antecederam a pesquisa.

Os dados obtidos por meio de formulários específicos foram: sociodemográficos, data do diagnóstico médico de DM2, condições clínicas, medicamentos prescritos para uso contínuo e resultado do teste laboratorial de hemoglobina glicada $(\mathrm{HbA1c})$ realizado no período de até seis meses anteriores à pesquisa. $\mathrm{Na}$ análise do controle glicêmico consideraram-se os seguintes parâmetros: $\mathrm{HbA} 1 \mathrm{c}$ menor que 7\% como controle glicêmico adequado e HbA1c maior ou igual a 7\% sendo o controle glicêmico inadequado 1 .

No cálculo do tamanho amostral considerou-se $90 \%$ a proporção de pessoas com DM2 que utilizavam medicamentos para o tratamento da doença ${ }^{6}$, erro amostral de $5 \%$ e nível de confiança de 95\%, obtendo-se 139 indivíduos. O número de participantes do estudo foi corrigido para 150, devido às possibilidades de perda e informações incompletas dos prontuários. A seleção da amostra ocorreu por aleatorização simples.

As informações foram armazenadas em um banco de dados com dupla conferência e analisadas por meio do software Stata ${ }^{\circledR}$ 12.1. Na descrição dos dados foram utilizadas medidas de tendência central e de dispersão para variáveis quantitativas e porcentagens para variáveis qualitativas. Para verificar as associações das variáveis categóricas empregou-se um modelo de regressão logística com estimativa de variância, sendo a Odds Ratio (OR) a medida de efeito analisada com seus respectivos intervalos de confiança de 95\% (IC95\%).

O presente estudo foi aprovado pelo Comitê de Ética em Pesquisa local com obtenção do Certificado de Apresentação para Apreciação Ética número 08738212.9.0000.5426. 


\section{RESULTADOS}

Nesta pesquisa foram revisados 198 prontuários, sendo que, 150 foram incluídos e 48 excluídos. As exclusões ocorreram por: informações incompletas acerca do tratamento medicamentoso (62\%), ausência de resultado do teste laboratorial de $\mathrm{HbA} 1 \mathrm{c}$ realizado no período de até seis meses anteriores à pesquisa (33\%) e prontuários de pessoas com diabetes mellitus tipo 1 (5\%).

$\mathrm{Na}$ amostra estudada predominou o sexo feminino $(61,3 \%)$. A idade dos participantes variou de 35 a 99 anos, com média de 66,4 14 anos. O tempo médio de diagnóstico médico do DM2 foi de 9,2 $\pm 9,2$ anos. O controle glicêmico apresentou-se inadequado em $75,3 \%$ das pessoas estudadas e a média de hemoglobina glicada de $8,8 \pm 2,4 \%$ (Tabela 1).

O número de medicamentos prescritos variou 1 a 14 , com média de $6,4 \pm 2,8$. Já o número de medicamentos prescritos para o tratamento do DM2 foi de 1 a 3, com

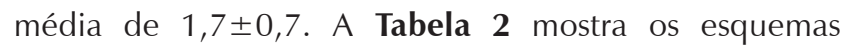
farmacoterapêuticos conforme os grupos etários. Nas pessoas com idade inferior a 60 anos predominou a prescrição de sulfonilureias ( $77 \%$ de glibenclamida e $23 \%$ gliclazida). Dentre os idosos, predominou a prescrição de biguanida (metformina), seguido de sulfonilureias (85\% de gliclazida de e $15 \%$ de glibenclamida) e insulina, respectivamente.

Verificou-se que o controle glicêmico não esteve associado a nenhum sexo, grupo etário, bem como, ao tempo de diagnóstico do DM2. Contudo, percebeu-se associação entre o controle glicêmico inadequado e o uso de terapia oral combinada $[\mathrm{OR}=0,32$ (IC95\% 0,12-0,80)] e com insulinoterapia isolada ou em associação com antidiabético oral (ADO) $[\mathrm{OR}=0,05$ (IC95\% 0,01-0,18)]. Além disso, evidenciou-se associação entre o controle glicêmico inadequado e a prescrição de dois ou três medicamentos (Tabela 3).

Tabela 1. Caracterização da amostra estudada.

\begin{tabular}{lcc}
\hline Variável & Frequência Absoluta (n) & Frequência Relativa (\%) \\
Sexo & & \\
Feminino & 92 & 61,3 \\
Masculino & 58 & 38,7 \\
Grupo etário (anos) & 4 & 2,7 \\
$30-39$ & 20 & 13,3 \\
$40-49$ & 26 & 17,3 \\
$50-59$ & 33 & 22,0 \\
$60-69$ & 34 & 22,7 \\
$70-79$ & 30 & 20,0 \\
$80-89$ & 3 & 2,0 \\
$90-99$ & & \\
Tempo de diagnóstico do diabetes mellitus tipo 2 (anos) & 31 & 20,7 \\
$<5$ & 25 & 16,7 \\
$5-9$ & 42 & 28,0 \\
$10-14$ & 52 & 34,6 \\
$\geq 15$ & & \\
Controle glicêmico & 113 & 75,3 \\
Inadequado (HbA1c $\geq 7 \%)$ & 37 & 24,7 \\
Adequado (HbA1c $<7 \%)$ & & \\
\hline
\end{tabular}

Tabela 2. Medicamentos prescritos para o tratamento do diabetes mellitus tipo 2 por grupo etário.

\begin{tabular}{ccccccc}
\hline $\begin{array}{c}\text { Grupo etário } \\
\text { (anos) }\end{array}$ & $\begin{array}{c}\text { Apenas metformina } \\
\mathbf{n}(\%)\end{array}$ & $\begin{array}{c}\text { Apenas sulfonilureia } \\
\mathbf{n}(\%)\end{array}$ & $\begin{array}{c}\text { Apenas insulina } \\
\mathbf{n}(\%)\end{array}$ & $\begin{array}{c}\text { Metformina+sulfonilureia } \\
\mathbf{n}(\%)\end{array}$ & $\begin{array}{c}\text { ADO*+insulina } \\
\mathbf{n}(\%)\end{array}$ & $\begin{array}{c}\text { Outros } \\
\mathbf{n}(\%)\end{array}$ \\
\hline $30-39$ & $1(25)$ & $0(0)$ & $2(50)$ & $0(0)$ & $0(25)$ & $0(0)$ \\
$40-49$ & $6(30)$ & $2(10)$ & $2(10)$ & $5(25)$ & $0(0)$ & $0(0)$ \\
$50-59$ & $5(19,2)$ & $17(65,4)$ & $4(15,4)$ & $9(27,3)$ & $12(36,4)$ & $1(3)$ \\
$60-69$ & $10(30,3)$ & $0(0)$ & $1(3)$ & $0(0)$ & $0(0)$ & $0(0)$ \\
$70-79$ & $14(41,2)$ & $18(52,9)$ & $2(5,9)$ & $0(0)$ & $0(0)$ & $0(0)$ \\
$80-89$ & $18(60)$ & $7(23,3)$ & $5(16,7)$ & $0(0)$ & $0(0)$ & $0(0)$ \\
$90-99$ & $0(0)$ & $0(0)$ & $3(100)$ & $0(0)$ & $0(0)$ \\
\hline
\end{tabular}

*ADO: antidiabético oral. 
Tabela 3. Associação do controle glicêmico segundo as variáveis sociodemográficas, clínicas e farmacoterapêuticas.

\begin{tabular}{|c|c|c|c|c|}
\hline \multirow[b]{2}{*}{ Variável } & \multirow[b]{2}{*}{ Amostra total } & \multicolumn{2}{|c|}{ Controle glicêmico } & \multirow{2}{*}{$\begin{array}{c}\text { OR } \\
\text { (IC95\%) }\end{array}$} \\
\hline & & $\begin{array}{l}\text { Inadequado } \\
\text { (HbA1c } \geq 7 \%)\end{array}$ & $\begin{array}{c}\text { Adequado } \\
(\text { HbA1c }<7 \%)\end{array}$ & \\
\hline \multicolumn{5}{|l|}{ Gênero } \\
\hline Masculino & $58(38,7)$ & $47(81)$ & 11(19) & Referência \\
\hline Feminino & $92(61,3)$ & $66(71,7)$ & $26(28,3)$ & $1,68(0,75 ; 3,73)$ \\
\hline \multicolumn{5}{|l|}{ Grupo etário (anos) } \\
\hline $30-39$ & $4(2,7)$ & $3(75)$ & $1(25)$ & Referência \\
\hline $40-49$ & $20(13,3)$ & $17(85)$ & $3(15)$ & $0,52(0,04 ; 6,94)$ \\
\hline $50-59$ & $26(17,3)$ & $22(84,6)$ & $4(15,4)$ & $0,54(0,04 ; 6,65)$ \\
\hline $60-69$ & $33(22,0)$ & $24(72,7)$ & $9(27,3)$ & $1,12(0,10 ; 12,26)$ \\
\hline $70-79$ & $34(22,7)$ & $20(58,8)$ & $14(41,2)$ & $2,1(0,19 ; 22,32)$ \\
\hline 80-89 & $30(20,0)$ & $25(83,3)$ & $5(16,7)$ & $0,6(0,05 ; 7,01)$ \\
\hline $90-99$ & $3(2,0)$ & $2(66,7)$ & $1(33,3)$ & $1,5(0,05 ; 40,63)$ \\
\hline \multicolumn{5}{|c|}{ Tempo de diagnóstico do diabetes mellitus tipo 2 (anos) } \\
\hline$<5$ & $31(20,7)$ & $24(77,4)$ & $7(22,6)$ & Referência \\
\hline $5-9$ & $25(16,7)$ & $20(80)$ & $5(20)$ & $0,85(0,23 ; 3,12)$ \\
\hline $10-14$ & $42(28,0)$ & $31(73,8)$ & $11(26,2)$ & $1,21(0,41 ; 3,60)$ \\
\hline$\geq 15$ & $52(34,6)$ & $38(73,1)$ & $14(26,9)$ & $1,26(0,44 ; 3,57)$ \\
\hline \multicolumn{5}{|l|}{ Tratamento medicamentoso } \\
\hline Monoterapia oral & $48(32)$ & $24(50)$ & $24(50)$ & Referência \\
\hline Terapia oral Combinada & $41(27,3)$ & $31(75,6)$ & $10(24,4)$ & $0,32(0,12 ; 0,80)$ \\
\hline Insulinoterapia com ou sem ADO* & $61(40,7)$ & $58(95,1)$ & $3(4,9)$ & $0,05(0,01 ; 0,18)$ \\
\hline \multicolumn{5}{|c|}{ Número de medicamentos para o tratamento do DM2 } \\
\hline 1 & $60(40)$ & $36(60)$ & $24(40)$ & Referência \\
\hline 2 & $72(48)$ & $60(83,3)$ & $12(16,7)$ & $0,3(0,13 ; 0,67)$ \\
\hline 3 & $18(12)$ & $17(94,4)$ & $1(5,6)$ & $0,08(0,01 ; 0,70)$ \\
\hline
\end{tabular}

*ADO: antidiabético oral.

\section{DISCUSSÃO}

Neste estudo, a amostra foi predominantemente do sexo feminino, idade igual ou superior a 60 anos, tempo médio de diagnóstico da doença próximo a dez anos, controle glicêmico inadequado. A prescrição de medicamentos em monoterapia foi a mais frequente, entretanto, chamou atenção o uso de sulfonilureia como monoterapia, particularmente entre os idosos.

Dados do Sistema de Informação da Atenção Básica do período estudado mostrou que 58\% das pessoas com DM2 cadastradas nas USFs participantes da pesquisa eram do sexo feminino e $70 \%$ idosos(as) ${ }^{7}$. Desta maneira, podemos inferir que a amostra estudada representa a população das USFs nos aspectos relacionados ao sexo e grupos etários.

O tempo de diagnóstico do DM2 igual ou superior a 15 anos obteve maior frequência, o que reflete serem os idosos a maioria dos participantes ${ }^{8}$. Verificou-se elevada proporção de participantes do estudo com controle glicêmico inadequado. Dado também observado em um estudo realizado na ESF de Pernambuco ${ }^{9}$. Isso é preocupante, uma vez que, o controle glicêmico inadequado nas USFs pode demandar utilização de outros serviços da rede de atenção à saúde, com aumento dos custos e hospitalizações. Logo, sugere-se a elaboração de planos terapêuticos singulares e ações de educação coletivas para otimizar o controle do DM24,10.

Os medicamentos são essenciais para o controle do DM21,2,11. Existem diversos fatores que influenciam na farmacoterapia, destacam-se as características fisiopatológicas da doença em um determinado tempo, bem como, aquelas relacionadas aos indivíduos, mecanismo de ação dos fármacos, reações adversas a medicamentos (RAMs), acesso aos medicamentos prescritos, adesão, dentre outros ${ }^{12-13}$. Nesta pesquisa, a metformina em monoterapia ou terapia combinada foi prescrita em todos os grupos etários. A diarreia é uma RAM comum e descrita na literatura ao uso de metformina e que dificulta a adesão $\mathrm{O}^{14-15}$. Contudo, neste estudo não foram analisados os dados referentes às RAMs devido à frequente falta desta informação nos prontuários estudados. Outra classe de medicamentos prescritos foram as sulfonilureias, as quais são secretagogas e que não deveriam ser a primeira opção no tratamento do DM2. Verificou-se 
elevada frequência da prescrição destes medicamentos em idosos, especificamente glibenclamida. A literatura considera inapropriado este medicamento para idosos, uma vez que, pode levar a hipoglicemia ${ }^{16}$. Assim, propõe-se a educação continuada dos prescritores acerca deste tema.

Evidenciou-se associação entre controle glicêmico inadequado com terapia oral combinada e também insulinoterapia com ou sem ADO. Além disso, dois ou três medicamentos prescritos estiveram associados ao controle glicêmico inadequado. Estes resultados corroboram com estudos encontrados na literatura ${ }^{12-15}$. Ressalta-se a importância da atuação multiprofissional no tratamento medicamentoso do DM2. A equipe de saúde deve ser responsável pelo olhar cuidadoso aos usuários de medicamentos nas USFs' ${ }^{17-19}$. O farmacêutico tem-se apresentado um profissional fundamental às esquipes da Atenção Primária à Saúde para o cuidado as DCNT, dentre elas o DM2. Um estudo clínico randomizado realizado em seis Unidades Básicas de Saúde de Ouro Preto, Minas Gerais mostrou que usuários acompanhados pelos serviços de atenção farmacêutica apresentaram redução significativa dos níveis de hemoglobina glicada quando comparados ao grupo controle ${ }^{20}$.

A principal limitação da pesquisa foi analisar apenas as informações obtidas por meio dos prontuários. Percebeu-se a carência de informações documentadas relacionadas ao tratamento nutricional, exercícios físicos, bem como, relatos sobre as RAMs, adesão e acesso aos medicamentos prescritos.

Este estudo evidenciou o controle glicêmico inadequado na maioria dos participantes. A monoterapia foi o tratamento mais frequente, embora, uma parcela considerável estudada usasse sulfonilureia em monoterapia, o que está em desacordo com as atuais diretrizes preconizadas para o tratamento do DM2. Este achado destaca a necessidade de capacitação das equipes das USFs em relação à farmacoterapia do DM2.

\section{REFERÊNCIAS}

1. Sociedade Brasileira de Diabetes. Diretrizes da Sociedade Brasileira de Diabetes 2015-2016. Rio de Janeiro: AC Farmacêutica; 2016.

2. American Diabetes Association. Standarts of Medical Care in Diabetes - 2017. Diabetes Care. 2017;40(1):S11-S25.

3. Brasil. Ministério da Saúde. Vigitel Brasil 2016. Brasília: Ministério da Saúde; 2017.

4. Brasil. Ministério da Saúde. Estratégias para o cuidado da pessoa com doença crônica: diabetes mellitus. Brasília: Ministério da Saúde; 2017.

5. Pereira SEA, Costa D, Penido R, Batista ANS, Calheiros A, Ferreira GV, Tavares JW, Marins RB, Messias YJ. Fatores de risco e complicações de doenças crônicas não transmissíveis. Ciênc Saúde (Porto Alegre). 2017;10(4):213-9. https://doi.org/10.15448/1983652X.2017.4.26446

6. Oliveira MA, Luiza VL, Tavares NUL, Mengue SS, Arrais PSD, Faria MR, Pizzol TSD, Ramos LR, Bertoldi AD. Acesso a medicamentos para doenças crônicas no Brasil: uma abordagem multidimensional. Rev Saúde Pública. 2016;50(Suppl 2):6s. http://dx.doi.org/10.1590/ s1518-8787.2016050006161

7. Prefeitura Municipal de Ribeirão Preto. Secretaria da Saúde. Plano Municipal de Saúde 2014-2017. Ribeirão Preto: Prefeitura Municipal de Ribeirão Preto; 2013.

8. Silva LMC, Palha PF, Barbosa GR, Protti ST, Ramos AS. Aposentados com diabetes tipo 2 na Saúde da Família em Ribeirão Preto, São Paulo - Brasil. Rev Esc Enferm USP. 2010;44(2):462-8. https://doi. org/10.1590/S0080-62342010000200031

9. Lima RF, Fontbonne A, Carvalho EMF, Montarroyos UR, Barreto MNSC, Cesse EAP. Factors associated with glycemic control in people with diabetes at the family health strategy in Pernambuco. Rev Esc Enferm USP. 2016; 50(6):937-45. https://doi.org/10.1590/ s0080-623420160000700009

10. Salci MA, Meirelles BHS, Silva DMGV. Primary care for diabetes mellitus patients from the perspective of the care model for chronic conditions. Rev Latino-Am Enfermagem. 2017;25:e2882. https:// doi.org/10.1590/1518-8345.1474.2882

11. Alves da Conceição R, Nogueira da Silva P, Barbosa MLC. Fármacos para o tratamento do diabetes tipo II: uma vista ao passado e um olhar para o futuro. Rev Virtual Quim. 2017;9(2):514-34.

12. Brummel A, Carlson AM. Comprehensive medication management and medication adherence for chronic conditions. J Manag Care Spec Pharm. 2016;22(1):56-62. https://doi.org/10.18553/ jmcp.2016.22.1.56

13. Costa SS, Rosales RA, Ávila JÁ, Pelzer MT, Lange C. Adesão de idosos com diabetes mellitus à terapêutica: revisão integrativa. Cogitare Enferm. 2017;22(3):e47720. https://doi.org/10.5380/ ce.v22i3.47720

14. May M, Schindler C. Clinically and pharmacologically relevant interactions of antidiabetic drugs. Ther Adv Endocrinol Metab. 2016;7(2):69-83. https://doi.org/10.1177/2042018816638050

15. Maruthur NM, Tseng E, Hutfless S, Wilson LM, Suarez-Cuervo C, Berger Z, Chu Y, lyoha E, Segal JB, Bolen S. Diabetes medications as monotherapy or metformin-based combination therapy for type 2 diabetes: a systematic review and meta-analysis. Ann Intern Med. 2016;164(11):740-51. https://doi.org/10.7326/M15-2650

16. American Geriatrics Society. American Geriatrics Society 2015 Updated Beers Criteria for Potentially Inappropriate Medication Use in Older Adults. J Am Geriatr Soc. 2015;63(11):2227-46. https://doi. org/10.1111/jgs.13702

17. Cunha GT, Campos GWS. Apoio matricial e atenção primária em saúde. Saúde Soc. 2011;20(4):961-70. https://doi.org/10.1590/ S0104-12902011000400013

18. Grillo MFF. Efeito de diferentes modalidades de educação para o autocuidado a pacientes com diabetes. Rev Assoc Med Bras. 2013;59(4):400-5. https://doi.org/10.1016/j.ramb.2013.02.006

19. Malta DC, Silva Júnior JB. Plano de ações estratégicas para o enfrentamento das doenças crônicas não transmissíveis no Brasil após 3 anos de implantação, 2011-2013. Epidemiol Serv Saúde. 2014;23(3):389-95. https://doi.org/10.5123/S167949742014000300002

20. Mourão AO, Ferreira WR, Martins MA, Reis AM, Carrillo MR, Guimarães AG, Ev LS. Pharmaceutical care program for type 2 diabetes patients in Brazil: a randomised controlled trial. Int J Clin Pharm. 2013;35(1):79-86 https://doi.org/10.1007/s11096-0129710-7 